\title{
Uso de conos de metal trabecular para defectos óseos severos en cirugía de revisión de reemplazo total de rodilla
}

\author{
Ignacio García-Mansilla, Julián Costantini, Tomás Nicolino, Juan Astoul Bonorino, Lisandro Carbó \\ Sector Artroscopia y Prótesis de Rodilla, Hospital Italiano de Buenos Aires, Ciudad Autónoma de Buenos Aires, Argentina
}

\begin{abstract}
RESUMEN
Introducción: Las alternativas reconstructivas para defectos óseos severos en la cirugía de revisión de prótesis son las camisas metafisarias, los conos de metal trabecular y el injerto óseo impactado o estructural. El objetivo del estudio fue analizar la tasa de osteointegración de los conos de metal trabecular en pacientes con cirugía de revisión de prótesis total de rodilla. El objetivo secundario fue analizar los resultados funcionales, las tasas de complicaciones y reoperaciones. Materiales y Métodos: Cohorte retrospectiva de pacientes con conos de metal trabecular colocados en la cirugía de revisión de prótesis y un seguimiento mínimo de 2 años. Se evaluaron las causas de la revisión, cirugías previas, tipo de defecto óseo, cantidad y tipo de conos utilizados, y los diseños de las prótesis. Se realizó una evaluación clínico-radiográfica, se registraron las complicaciones y las revisiones ulteriores. Resultados: Se evaluó a 35 pacientes (49 conos de metal trabecular) en forma retrospectiva, con un seguimiento promedio de 32.1 meses. La mayoría de los defectos eran tibiales AORI 3, seguidos de los femorales tipo 3. La tasa de osteointegración de los conos fue del $94 \%$; la de complicaciones, del $20 \%$ y la de reoperaciones, del $8,5 \%$. El KSS objetivo promedio aumentó de 39 en el preoperatorio a 71 en el último control y el puntaje de la EAV promedio fue 8 y 2,5, respectivamente. Conclusión: La excelente tasa de osteointegración (94\%) y los buenos resultados clínicos posicionan a los conos de metal trabecular como una alternativa para los defectos óseos severos.
\end{abstract}

Palabras clave: Revisión; reemplazo total de rodilla; defectos óseos; prótesis total; rodilla, metal trabecular.

Nivel de Evidencia: IV

\section{The Use of Trabecular Metal Cones for the Management of Severe Bone Defects in Revision Total Knee Replacement}

\begin{abstract}
Introduction: Major bone defects represent a challenge during revision total knee arthroplasty (TKA) and there is still considerable debate about the best therapeutic option. The purpose of this study was to retrospectively assess the osseointegration rate of trabecular metal cones in revision TKA with severe bone defects. The secondary purpose was to evaluate the functional outcomes and complication and reoperation rates. Materials and Methods: A single-center, retrospective cohort including all consecutive cases of revision TKA using trabecular metal cones. All patients with a minimum 2-year follow-up were included in the study. Reasons for revision, number of previous surgeries, type of bone defect, and number and type of trabecular cones used were evaluated. Clinical and radiological outcomes were also analyzed as well as complications rates. Results: 35 patients (49 cones) were evaluated with a mean follow-up of 32.1 months (24-62). Most defects were localized in the tibia and were classified as AORI type 3. The rate of osseointegration of the cones was $94 \%$; the complication rate, $20 \%$; and the reoperation rate, $8.5 \%$. The mean KSS increased from 39 preoperatively to 71 at the last follow-up, and the mean VAS from 8 to 2.5. Conclusion: The excellent osseointegration rate $(94 \%)$, added to the good clinical outcomes, position the trabecular metal cones as an alternative to treat severe bone defects.
\end{abstract}

Keywords: Revision; total knee replacement; bone defects; knee; trabecular metal cones.

Level of Evidence: IV

\footnotetext{
Recibido el 9-6-2021. Aceptado luego de la evaluación el 23-6-2021 • Dr. IGNACIO GARCÍA-MANSILLA • ignaciogmansilla@gmail.com https://orcid.org/0000-0002-7247-3734 Cómo citar este artículo: García-Mansilla I, Costantini J, Nicolino TI, Astoul Bonorino J, Carbó L. Uso de conos de metal trabecular para defectos óseos severos en cirugía de revisión de reemplazo total de rodilla. Rev Asoc Argent Ortop Traumatol 2021;86(4):483-492. https://doi.org/10.15417/issn. 1852-7434.2021.86.4.1390
} 


\section{INTRODUCCIÓN}

Los defectos óseos severos continúan siendo un desafío en la revisión de una artroplastia de rodilla, principalmente los defectos AORI 2B y 3 que comprometen la estabilidad y la fijación del implante de revisión (Figura 1). ${ }^{1,2}$

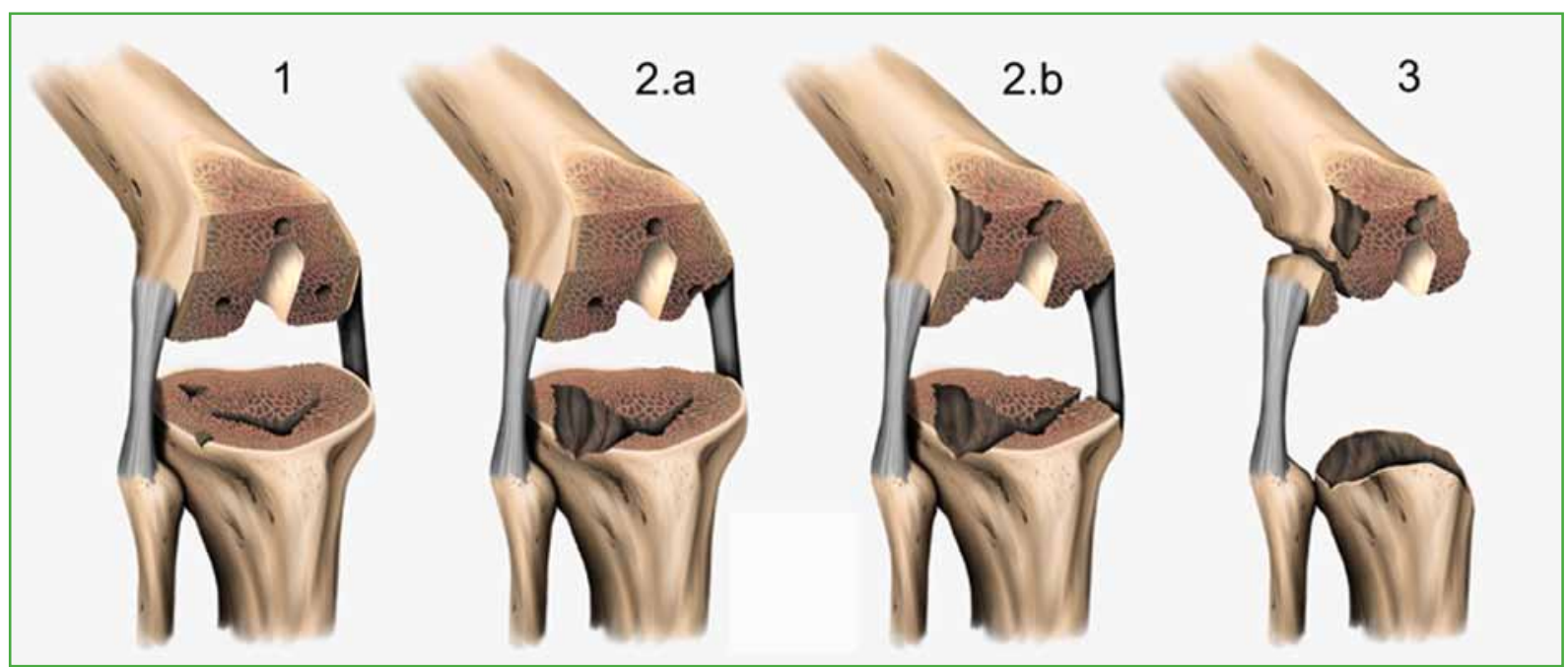

Figura 1. Clasificación de defectos óseos del Anderson Orthopaedic Research Institute (AORI). Tipo 1: Defectos menores femorales o tibiales con hueso metafisario intacto, que no comprometen la estabilidad del componente de revisión. Tipo 2: Hueso metafisario dañado, que requiere de una reconstrucción para proveer estabilidad al componente de revisión. Tipo 2A: Defectos en un cóndilo femoral o tibial. Tipo 2B: Defectos en ambos cóndilos femorales o tibiales.

Tipo 3: Segmento metafisario deficiente que compromete una porción importante de los cóndilos femorales o el platillo tibial, ocasionalmente asociados con lesión de ligamentos colaterales o tendón rotuliano.

Independientemente del sistema de reconstrucción utilizado, se ha demostrado que la fijación metafisaria es determinante para la supervivencia del implante. ${ }^{3-5}$ Las alternativas reconstructivas son, según el defecto, las camisas metafisarias, los conos de metal trabecular y el injerto óseo impactado o estructural.

El injerto óseo estructural tiene la desventaja de ser una opción técnicamente demandante para lograr una adecuada interfase injerto-huésped, y se han comunicado tasas de reabsorción y fallas mecánicas que ascienden al $23 \%$ a los 5 años. ${ }^{6}$ Por el contrario, los conos de metal trabecular son una opción versátil (diversos tamaños y formas compatibles con casi todos los sistemas de revisión) y proporcionan un soporte mecánico sin riesgo de reabsorción y colapso. ${ }^{7-12}$ Las desventajas son su elevado costo económico, no aportar stock óseo, la dificultad para extraerlos si hay una infección que lo requiera y ser altamente irritantes para los tejidos blandos circundantes.

En cuanto a las propiedades del material, el metal trabecular posee una estructura similar al hueso esponjoso, con una alta porosidad volumétrica (75-80\%), un bajo módulo de elasticidad ( $3 \mathrm{GPa}$ ) y alta fricción. Además, permiten la osteointegración a la vez que rellenan defectos óseos y presentan una tolerancia inmediata a cargas fisiológicas. Los defectos AORI 2B y 3 son su principal indicación y se han logrado resultados prometedores a mediano y largo plazo. ${ }^{13-21}$

El objetivo de este estudio fue analizar la tasa de osteointegración del implante en una serie de pacientes a quienes se les colocaron conos de metal trabecular en la cirugía de revisión de prótesis total de rodilla. Como objetivo secundario, se analizaron los resultados funcionales, las tasas de complicaciones y reoperaciones.

\section{MATERIALES Y MÉTODOS}

\section{Pacientes}

En este estudio retrospectivo, se incluyó, de forma consecutiva, a pacientes operados en nuestro Centro, en quienes se habían utilizado conos de metal trabecular en la cirugía de revisión de prótesis total de rodilla. Los criterios de inclusión fueron revisiones en uno o dos tiempos, por cualquier motivo, defectos óseos AORI 2B y 3, uso de uno o más conos de metal trabecular y un seguimiento mínimo de dos años. Se excluyó a aquellos pacientes con conos de metal trabecular en la cirugía primaria. 


\section{Métodos de evaluación}

Se tomaron radiografías posoperatorias inmediatas, a los 3 y 6 meses, al año de la cirugía, y en el último control para evaluar los signos de osteointegración y aflojamiento de los conos de metal trabecular o del implante. Se evaluaron los signos de radiolucidez progresiva u osteólisis alrededor de los componentes y en la interfase conohueso. La osteointegración radiográfica en el último control se definió como la ausencia de una línea radiolúcida entre el hueso y el metal trabecular.

Clínicamente los pacientes fueron evaluados antes de la cirugía y durante el seguimiento con el Knee Society Score (KSS) y la escala analógica visual para dolor. Se registraron las complicaciones intraoperatorias y posoperatorias, tempranas y tardías. Por último, se analizaron las tasas de reoperación y revisión (supervivencia del implante).

\section{Técnica quirúrgica}

Después del desbridamiento de los tejidos blandos, se retira el implante previo junto con el cemento. Se revalúa la extensión de la pérdida ósea, se clasifica el defecto remanente y se confirma la indicación de conos de metal trabecular. A continuación, se colocan los implantes de prueba para tamaño y forma, seleccionando el que proporcione el área más grande de contacto con el hueso (Figura 2).

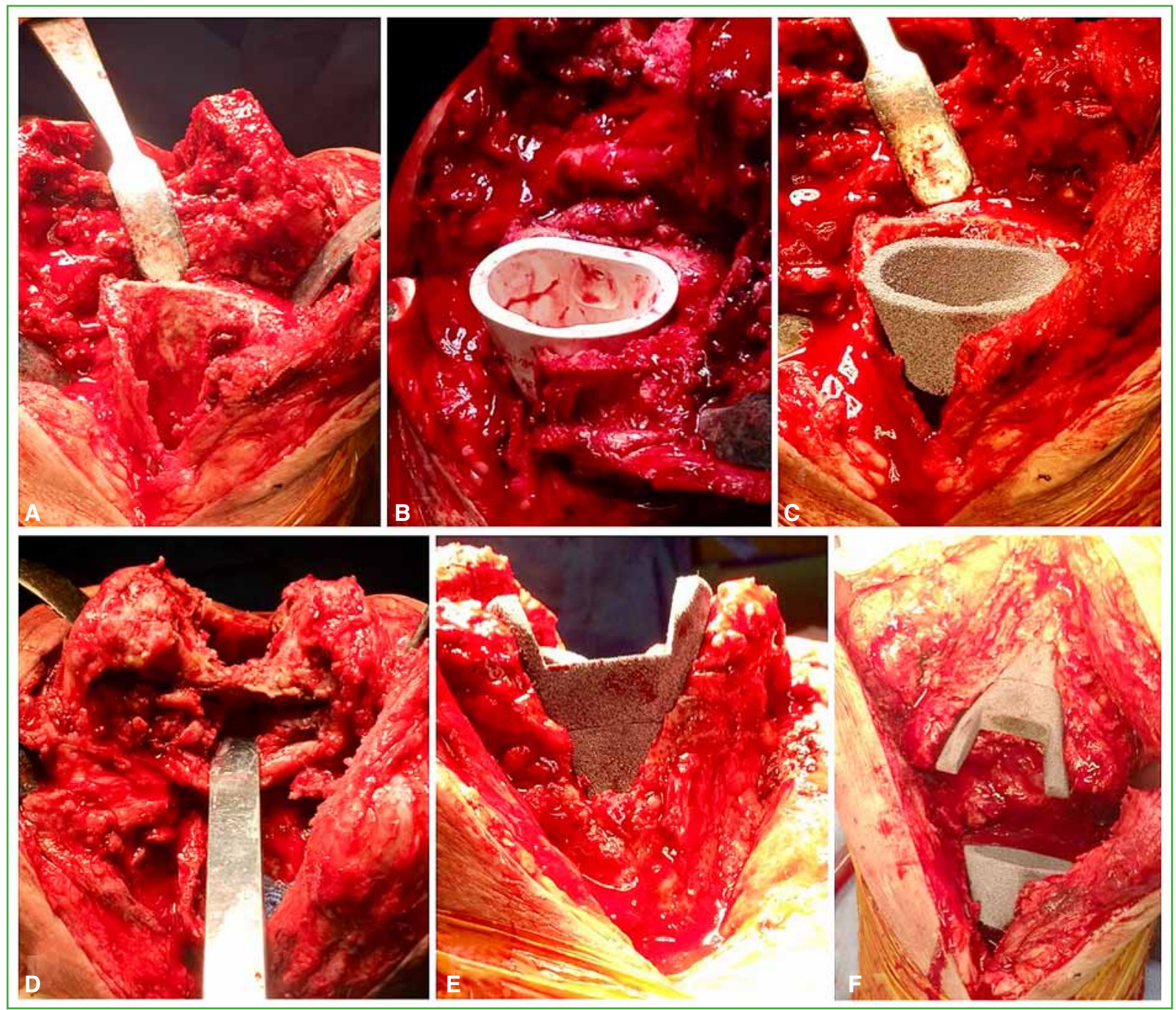

Figura 2. Imágenes intraoperatorias de la técnica quirúrgica. Se observa a nivel tibial un defecto metafisario no contenido luego de la extracción del implante y el desbridamiento del tejido no viable (A). Se coloca el implante de prueba de un cono tibial metafisario $(\mathbf{B})$ y luego se impacta el cono definitivo $(\mathbf{C})$. Debido a la magnitud del defecto remanente en el fémur (D), se decidió utilizar, en este caso, una combinación de un cono metafisario y uno diafisario (E y F). 
Después de la preparación del hueso huésped, se realiza la impactación del cono. El ajuste a presión del cono (press-fit) es de suma importancia para garantizar que el cemento no penetre entre el cono y el hueso. En el caso de combinar conos metafisarios y diafisarios, se recomienda utilizar cemento en la unión de ambos conos. Finalmente, los implantes definitivos se cementan en los conos, es recomendable también utilizar vástagos cementados. Las zonas expuestas de metal trabecular se recubren con cemento, ya que son altamente irritantes para las partes blandas (Figura 3).

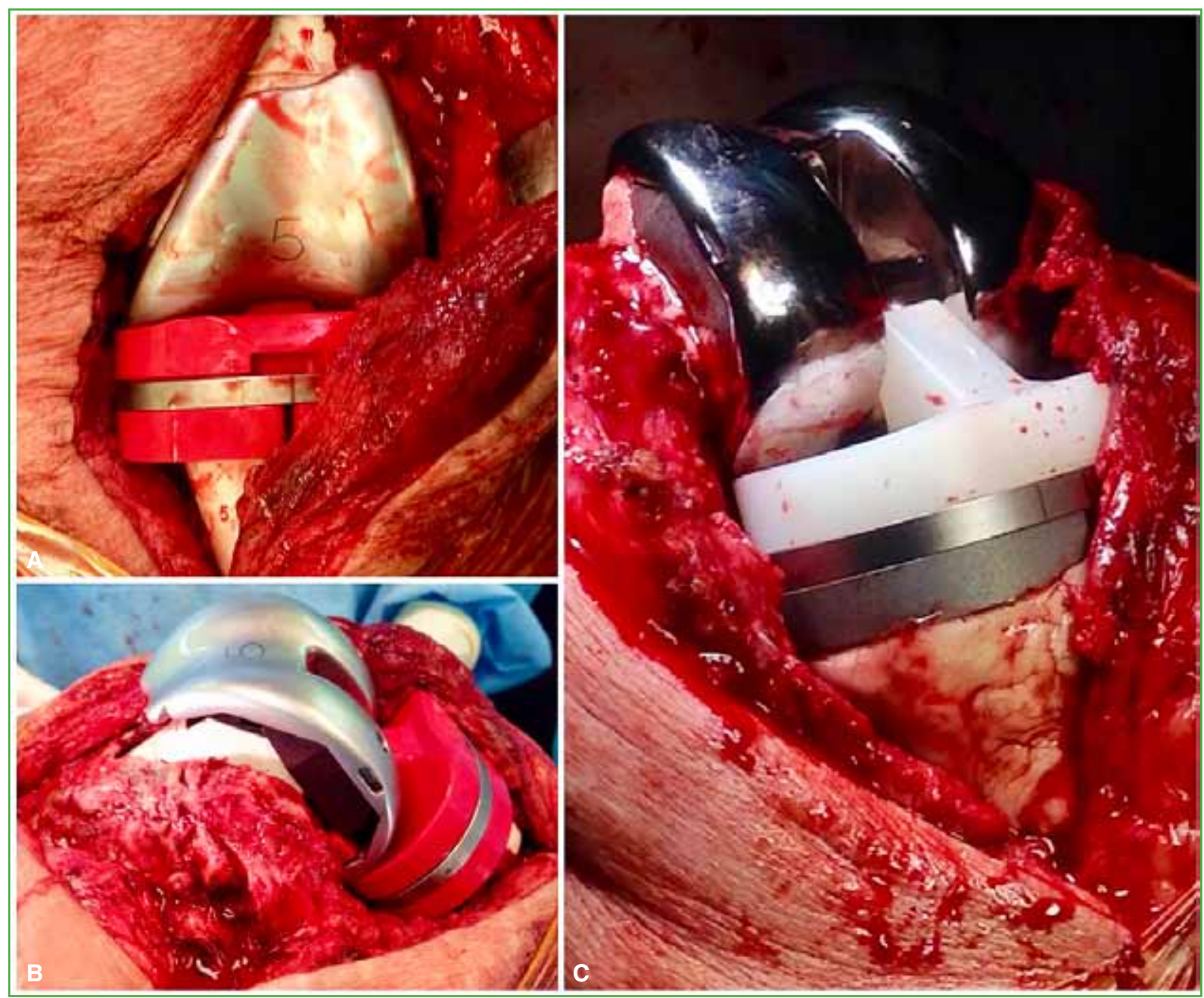

Figura 3. Continuación de las imágenes intraoperatorias de la Figura 2. Colocación de implantes de prueba (A y B) e implantes definitivos $(\mathbf{C})$. La zona expuesta del cono a nivel tibial se recubre con cemento para no irritar los tejidos blandos adyacentes $(\mathbf{C})$. 


\section{RESULTADOS}

Desde la incorporación de los conos de metal trabecular a fines de 2015, en nuestro Centro, se realizaron 215 revisiones de prótesis total de rodilla. En 45 (21\%) casos, se utilizaron conos de metal trabecular. Ocho pacientes fueron excluidos por no cumplir con el seguimiento mínimo y dos fallecieron antes de cumplir los dos años de seguimiento. Por lo tanto, nuestra serie quedó conformada por 35 pacientes, con un seguimiento promedio de 32.1 meses (rango 24-62). Ninguno se perdió durante el seguimiento. En la Tabla 1, se detallan las características de los pacientes y las causas de la revisión. La mayoría eran mujeres $(n=23 ; 6,7 \%)$, el implante previo más frecuente era una prótesis primaria $(\mathrm{n}=21,60 \%)$ y la causa de revisión más frecuente era el aflojamiento aséptico de una prótesis primaria $(\mathrm{n}=12 ; 34,2 \%)$.

Tabla 1. Características de los pacientes y causas de la revisión

Edad, años, promedio (rango)

$66.1(35-85)$

Índice de masa corporal, $\mathrm{kg} / \mathrm{m}^{2}$, promedio (rango)

Sexo, Femenino, n (\%)

$30,7(20-47)$

Cantidad de cirugías previas, promedio (rango)

$23(65,7 \%)$

$2,9(1-10)$

Implante previo

Prótesis primaria

$21(60 \%)$

Prótesis de revisión con vástagos

$12(34,2 \%)$

Prótesis modular

$1(2,8 \%)$

Aloprótesis

$1(2,8 \%)$

Causas de la revisión (n, \%)

Aflojamiento aséptico de prótesis primaria

$12(34,2 \%)$

Aflojamiento aséptico de prótesis de revisión

$6(17,1 \%)$

Aflojamiento séptico de prótesis primaria

$9(25,7 \%)$

Aflojamiento séptico de prótesis de revisión

$6(17,1 \%)$

Inestabilidad asociada a la prótesis

$1(2,8 \%)$

Prótesis dolorosa

$1(2,8 \%)$

En la Tabla 2, se muestra la distribución de los defectos óseos que requirieron relleno con conos de metal trabecular, clasificados según el AORI, ${ }^{1}$ y la localización y los tipos de conos utilizados.

Tabla 2. Defectos óseos que requirieron relleno con conos de metal trabecular clasificados según el AORI. Localización y tipo de conos utilizados durante la revisión

\begin{tabular}{|l|c|c|c|c|}
\hline & \multicolumn{3}{|c|}{ AORI } & \multicolumn{3}{c|}{ Conos } \\
\hline & 2B & $\mathbf{3}$ & Diafisarios & Metafisarios \\
\hline Fémur & 5 & 11 & 7 & 9 \\
\hline Tibia & 6 & 24 & 6 & 28 \\
\hline AORI = Anderson Orthopaedic Research Institute. & & & \\
\hline
\end{tabular}


En 10 casos, se usó una combinación de conos femorales y tibiales, y en tres casos, se combinaron conos metafisarios y diafisarios en un mismo paciente. En cuanto a los diseños de la prótesis para la revisión (Figura 4), más de la mitad fueron prótesis constreñidas $(\mathrm{n}=20 ; 57,1 \%)$, el 20\% fueron bisagras rotatorias $(\mathrm{n}=7)$; el 20\%, megaprótesis $(\mathrm{n}=7)$ y una de pivote medial $(2,8 \%)$. En dos casos, se usó, además, un injerto óseo impactado a nivel del fémur y, en cinco, se realizó una reconstrucción del aparato extensor (3 con aloinjerto y 2 con malla).
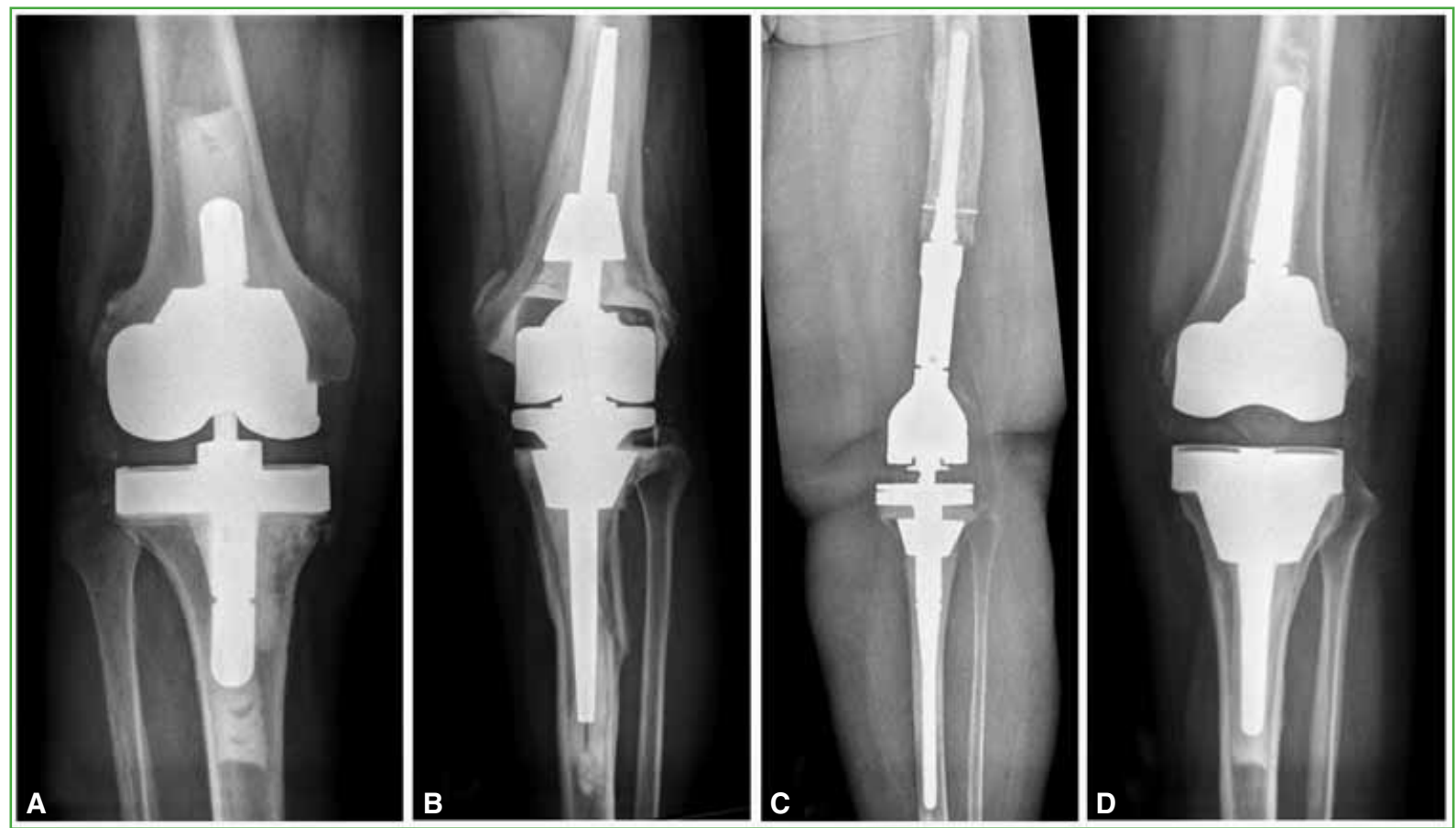

Figura 4. Diseños de prótesis utilizados en la revisión. A. Constreñida. B. Bisagra rotatoria. C. Megaprótesis. D. Pivote medial.

En cuanto a la evaluación radiográfica, la tasa de osteointegración de los conos de metal trabecular al final del seguimiento fue del $94 \%(\mathrm{n}=33)$. Un paciente tuvo un aflojamiento aséptico, por lo que se efectuó una revisión del implante a los dos años de la operación. Un paciente sufrió una infección temprana que se trató con una limpieza y retención del implante. La evolución fue desfavorable con aflojamiento del implante y se encuentra en plan de revisión. En el resto de la serie, hubo un paciente asintomático con imágenes radiolúcidas en el vástago femoral de una megaprótesis, sin signos de aflojamiento a nivel tibial donde se había colocado un cono de metal trabecular metafisario. La supervivencia sin revisión del cono de metal trabecular fue del 94\% en el último control. En la Figura 5, se muestran las estimaciones de supervivencia de Kaplan-Meier (revisión del cono como punto final) para todos los conos implantados $(\mathrm{n}=35)$.

La tasa de complicaciones fue del $20 \%(\mathrm{n}=7)$ y la de reoperaciones, del $8,5 \%(\mathrm{n}=3)$. Durante la cirugía, dos pacientes presentaron una fractura periprotésica incompleta de fémur (tratadas, de manera conservadora, con consolidación ósea en el seguimiento) (Figura 6). Se registraron cuatro complicaciones posoperatorias: un caso de rigidez, una luxación del aparato extensor (tratada de forma no quirúrgica), un tromboembolismo pulmonar, una infección temprana y un aflojamiento aséptico, ya mencionados. El paciente con rigidez posoperatoria fue tratado, con éxito, mediante una movilización bajo anestesia.

En cuanto a la evaluación clínica, el KSS promedio aumentó de 39 antes de la cirugía a 71 en el último control. El puntaje preoperatorio promedio de la escala analógica visual fue de 8 y de 2,5 en el último control. 


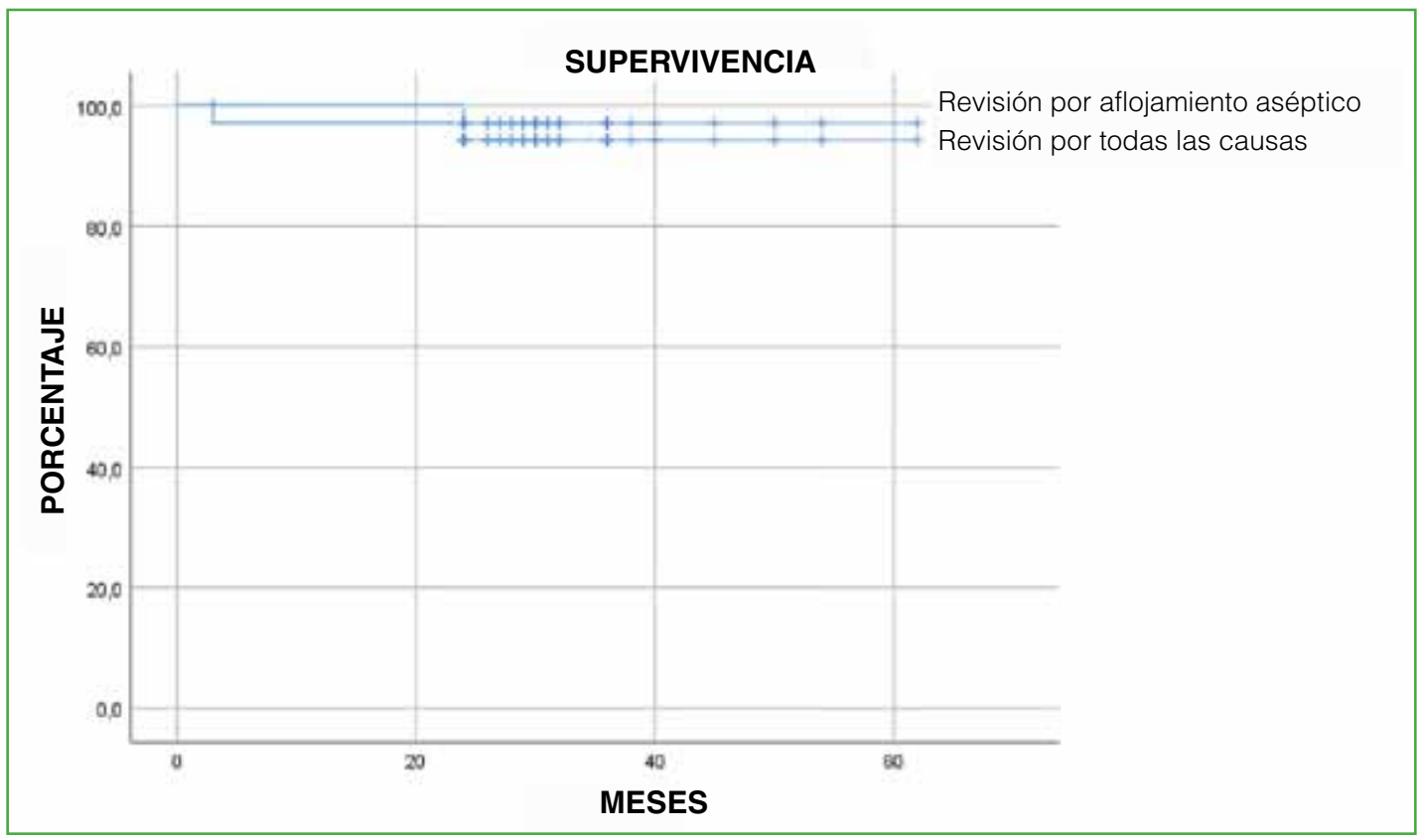

Figura 5. Curva de supervivencia de Kaplan-Meier. Tasas de supervivencia del $97 \%$ y $94 \%$ de los conos de metal trabecular tomando como punto de corte las revisiones por aflojamiento aséptico y las revisión por todas las causas, respectivamente.

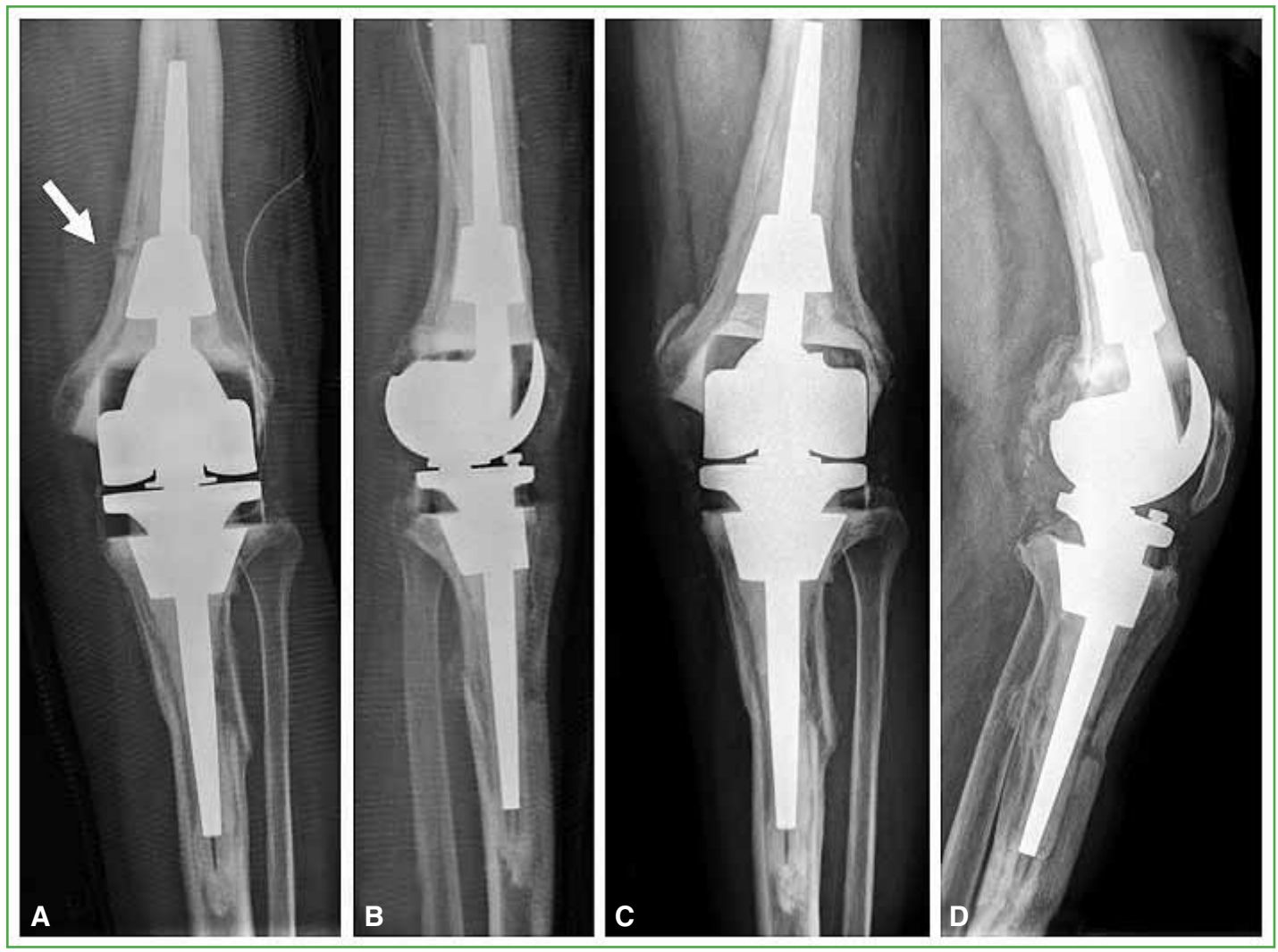

Figura 6. Radiografías posoperatorias inmediatas de frente (A) y de perfil (B) de rodilla. Se observa una fractura a nivel de la cortical medial del fémur (flecha blanca). C y D. Radiografías de control al año de la cirugía. Consolidación de la fractura. 


\section{DISCUSIÓN}

El tratamiento eficaz de los defectos óseos es fundamental para garantizar la estabilidad metafisaria del implante en casos de revisión de la prótesis total de rodilla., ${ }^{4,22}$ Como ya se comentó, las alternativas terapéuticas para los defectos óseos severos (AORI 2B y 3) son el injerto molido impactado, el aloinjerto estructural, las camisas metafisarias y los conos de metal trabecular. Estas técnicas están diseñadas para reconstruir la metáfisis femoral o tibial dañada y crear una plataforma estable para la fijación del implante.$^{2,3,23}$ El injerto óseo morcelizado impactado convierte el hueso femoral o tibial liso y esclerótico en una superficie apta para la interdigitación del cemento y la fijación del vástago. Esto es beneficioso en pacientes jóvenes, ya que es posible restaurar el stock óseo. Sin embargo, los defectos corticales periféricos son más difíciles de tratar con esta técnica, porque se requiere una malla metálica para contener el injerto. ${ }^{24,25}$ Los aloinjertos estructurales pueden sustituir los segmentos óseos deficientes tanto centrales como periféricos sin necesidad de una malla metálica adicional. A largo plazo, el aloinjerto estructural puede reabsorberse, fracturarse, infectarse o no consolidar. ${ }^{6,26} \mathrm{El}$ aumento de la tasa de fracaso de este tipo de reconstrucción estimuló el desarrollo de conos de metal trabecular altamente porosos que funcionan como alternativa a los aloinjertos estructurales. Estos dispositivos logran una sólida fijación metafisaria inicial y una eventual fijación biológica sin el riesgo de reabsorción o fractura del injerto., ${ }^{9,10,12,13}$ Por último, las camisas metafisarias tienen una forma escalonada y están recubiertas de perlas de titanio para producir una superficie porosa para el crecimiento óseo. El hueso huésped se prepara con una mecha y la camisa se une al componente femoral o tibial a través de un cono Morse.

Nuestra serie confirma los resultados ya publicados, a los 32 meses de seguimiento promedio (rango 24-62), la tasa de osteointegración es del 94\%, con un solo caso de falla mecánica. Las publicaciones a mediano plazo informan tasas de revisión por todas las causas del $8-15 \%{ }^{15,21}$ y tasas de revisión por reinfección del 5-19\%. ${ }^{13}$ En nuestra serie, la tasa de revisión por todas las causas fue del 6\% $(n=2)$ y del 3\% $(n=1)$ por reinfección. En 2016, Potter y cols. ${ }^{19}$ publicaron una serie de 159 conos de tantalio femorales en 157 pacientes y una tasa de supervivencia del $70 \%$ a los 5 años (incluye todas las causas de revisión). Se revisaron 23 conos, 14 por infección, seis por aflojamiento aséptico (todos en prótesis abisagradas con defectos tipo 3 en fémur) y tres por inestabilidad ligamentaria. De forma similar, en nuestra serie, el aflojamiento aséptico también se produjo en un caso de defecto AORI tipo 3 localizado en el fémur tratado con una prótesis de bisagra rotatoria.

$\mathrm{Al}$ ser una cirugía de rescate en pacientes complejos con múltiples cirugías previas, las complicaciones no son infrecuentes. Kamath y cols., ${ }^{21}$ en una serie de 63 pacientes tratados con conos tibiales (defectos 2A, 2B y 3) con un seguimiento mínimo de 5 años, comunicaron una tasa de complicaciones del 27\% (sépticas y asépticas). Por su parte, Brown y cols. ${ }^{14}$ tuvieron una tasa de complicaciones muy alta, del $45 \%$ en su serie de 83 pacientes. Las más frecuentes fueron infección profunda $(11,13 \%)$ y rigidez $(17,20 \%)$. En nuestra serie, la tasa de complicaciones fue del 20\% ( $\mathrm{n}=7$ ): un caso de infección posoperatoria temprana, un aflojamiento aséptico, dos fracturas intraoperatorias, un tromboembolismo pulmonar, un caso de rigidez y una luxación del aparato extensor.

Nuestro estudio tiene las limitaciones inherentes a los estudios retrospectivos, cuyos efectos son mitigados por la recolección prospectiva de los datos. La cantidad de pacientes podría considerarse baja; sin embargo, la cantidad incluida en las series publicadas varía desde $9^{27}$ hasta 157 en las series más extensas presentadas por la Mayo Clinic. ${ }^{19}$ El seguimiento es corto debido a la reciente incorporación de estos implantes en nuestro medio, un mayor seguimiento de nuestra población proporcionará información adicional. Además, esta serie incluye tanto pacientes con aflojamientos sépticos como asépticos, diversos tipos de implante e incluso pacientes con reconstrucciones del aparato extensor. Esta heterogeneidad podría dificultar la interpretación de los resultados.

La excelente tasa de osteointegración (94\%), sumada a los buenos resultados clínicos, posicionan a los conos de metal trabecular como una alternativa en el tratamiento de los defectos óseos metafisarios severos. Se requieren análisis a largo plazo y la comparación con otras opciones de reconstrucción para determinar si este método de reconstrucción proporcionará un éxito clínico superior a largo plazo.

Conflicto de intereses: Los autores no declaran conflictos de intereses.

ORCID de J. Costantini: https://orcid.org/0000-0001-8900-6254 ORCID de T. I. Nicolino: https://orcid. org/0000-0002-9550-3713
ORCID de J. Astoul Bonorino: https://orcid.org/0000-0001-6798-8242 ORCID de L. Carbó: https:///rcid. org/0000-0002-8053-0890 


\section{BIBLIOGRAFÍA}

1. Engh GA, Ammeen DJ. Classification and preoperative radiographic evaluation: knee. Orthop Clin North Am 1998;29(2):205-17. https://doi.org/10.1016/S0030-5898(05)70319-9

2. Lei P, Hu R, Hu Y. Bone defects in revision total knee arthroplasty and management: bone defects in revision TKA. Orthop Surg 2019;11(1):15-24. https://doi.org/10.1111/os.12425

3. Sculco PK, Abdel MP, Hanssen AD, Lewallen DG. The management of bone loss in revision total knee arthroplasty: rebuild, reinforce, and augment. Bone Joint J 2016;98-B(1_Supple_A):120-4. https://doi.org/10.1302/0301-620X.98B1.36345

4. Haidukewych GJ, Hanssen A, Dickey Jones R. Metaphyseal fixation in revision total knee arthroplasty: indications and techniques: Am Acad Orthop Surg 2011;19(6):311-8. https://doi.org/10.5435/00124635-201106000-00001

5. Ayerza M, Yacuzzi C, Costa Paz M, Aponte Tinao L, Makino A, Múscolo DL. Cirugía de revisión protésica en pacientes con defectos óseos masivos de la rodilla. Rev Artrosc 2007;14(1):34-9. Disponible en: https://www. revistaartroscopia.com/ediciones-anteriores/2007/volumen-14-numero-1/35-volumen-05-numero-1/volumen-14numero-1/618-cirugia-de-revision-protesica-en-pacientes-con-defectos-oseos-masivos-de-la-rodilla

6. Bauman RD, Lewallen DG, Hanssen AD. Limitations of structural allograft in revision total knee arthroplasty. Clin Orthop 2009;467(3):818-24. https://doi.org/10.1007/s11999-008-0679-4

7. Meneghini RM, Lewallen DG, Hanssen AD. Use of porous tantalum metaphyseal cones for severe tibial bone loss during revision total knee replacement: J Bone Joint Surg Am 2009;91(Suppl 2):131-8. https://doi.org/10.2106/JBJS.H.01061

8. Boureau F, Putman S, Arnould A, Dereudre G, Migaud H, Pasquier G. Tantalum cones and bone defects in revision total knee arthroplasty. Orthop Traumatol Surg Res 2015;101(2):251-5. https://doi.org/10.1016/j.otsr.2014.11.020

9. Divano S, Cavagnaro L, Zanirato A, Basso M, Felli L, Formica M. Porous metal cones: gold standard for massive bone loss in complex revision knee arthroplasty? A systematic review of current literature. Arch Orthop Trauma Surg 2018;138(6):851-63. https://doi.org/10.1007/s00402-018-2936-7

10. Bonanzinga T, Gehrke T, Zahar A, Zaffagnini S, Marcacci M, Haasper C. Are trabecular metal cones a valid option to treat metaphyseal bone defects in complex primary and revision knee arthroplasty? Joints 2018;06(01):058-64. https://doi.org/10.1055/s-0037-1608950

11. Jacquet C, Ros F, Guy S, Parratte S, Ollivier M, Argenson J-N. Trabecular metal cones combined with short cemented stem allow favorable outcomes in aseptic revision total knee arthroplasty. J Arthroplasty 2021;36(2):65763. https://doi.org/10.1016/j.arth.2020.08.058

12. Roach RP, Clair AJ, Behery OA, Thakkar SC, Iorio R, Deshmukh AJ. Aseptic loosening of porous metaphyseal sleeves and tantalum cones in revision total knee arthroplasty: a systematic review. J Knee Surg 2020 Feb 19. https://doi.org/10.1055/s-0040-1701434

13. Kim E, Patel N, Chughtai M, Elmallah RDK, Delanois RE, Harwin SF, et al. Tantalum cones in revision total knee arthroplasty. J Knee Surg 2016;29(08):621-6. https://doi.org/10.1055/s-0036-1593370

14. Brown NM, Bell JA, Jung EK, Sporer SM, Paprosky WG, Levine BR. The use of trabecular metal cones in complex primary and revision total knee arthroplasty. J Arthroplasty 2015;30(9):90-3. https://doi.org/10.1016/j.arth.2015.02.048

15. De Martino I, De Santis V, Sculco PK, D’Apolito R, Assini JB, Gasparini G. Tantalum cones provide durable midterm fixation in revision TKA. Clin Orthop Relat Res 2015;473(10):3176-82. https://doi.org/10.1007/s11999-015-4338-2

16. Abdelaziz H, Jaramillo R, Gehrke T, Ohlmeier M, Citak M. Clinical survivorship of aseptic revision total knee arthroplasty using hinged knees and tantalum cones at minimum 10-year follow-up. J Arthroplasty 2019;34(12):3018-22. https://doi.org/10.1016/j.arth.2019.06.057

17. Abdelaziz H, Biewald P, Anastasiadis Z, Haasper C, Gehrke T, Hawi N, et al. Midterm results after tantalum cones in 1-stage knee exchange for periprosthetic joint infection: a single-center study. J Arthroplasty 2020;35(4):1084-9. https://doi.org/10.1016/j.arth.2019.11.016

18. Burastero G, Cavagnaro L, Chiarlone F, Alessio-Mazzola M, Carrega G, Felli L. The use of tantalum metaphyseal cones for the management of severe bone defects in septic knee revision. J Arthroplasty 2018;33(12):3739-45. https://doi.org/10.1016/j.arth.2018.08.026

19. Potter GD, Abdel MP, Lewallen DG, Hanssen AD. Midterm results of porous tantalum femoral cones in revision total knee arthroplasty: J Bone Joint Surg 2016;98(15):1286-91. https://doi.org/10.2106/JBJS.15.00874 
20. Girerd D, Parratte S, Lunebourg A, Boureau F, Ollivier M, Pasquier G, et al. Total knee arthroplasty revision with trabecular tantalum cones: Preliminary retrospective study of 51 patients from two centres with a minimal 2-year follow-up. Orthop Traumatol Surg Res 2016;102(4):429-33. https://doi.org/10.1016/j.otsr.2016.02.010

21. Kamath AF, Lewallen DG, Hanssen AD. Porous tantalum metaphyseal cones for severe tibial bone loss in revision knee arthroplasty: a five to nine-year follow-up. J Bone Joint Surg Am 2015;97(3):216-23. https://doi.org/10.2106/JBJS.N.00540

22. Ponzio DY, Austin MS. Metaphyseal bone loss in revision knee arthroplasty. Curr Rev Musculoskelet Med 2015;8(4):361-7. https://doi.org/10.1007/s12178-015-9291-x

23. Beckmann NA, Mueller S, Gondan M, Jaeger S, Reiner T, Bitsch RG. Treatment of severe bone defects during revision total knee arthroplasty with structural allografts and porous metal cones-A systematic review. $J$ Arthroplasty 2015;30(2):249-53. https://doi.org/10.1016/j.arth.2014.09.016

24. Lotke PA, Carolan GF, Puri N. Impaction grafting for bone defects in revision total knee arthroplasty. Clin Orthop 2006;446:99-103. https://doi.org/10.1097/01.blo.0000214414.06464.00

25. Hilgen V, Citak M, Vettorazzi E, Haasper C, Day K, Amling M, et al. 10-year results following impaction bone grafting of major bone defects in 29 rotational and hinged knee revision arthroplasties: A follow-up of a previous report. Acta Orthop 2013;84(4):387-91. https://doi.org/10.3109/17453674.2013.814012

26. Clatworthy MG, Ballance J, Brick GW, Chandler HP, Gross AE. The use of structural allograft for uncontained defects in revision total knee arthroplasty: a minimum five-year review. J Bone Joint Surg Am 2001;83(3):404-11. https://doi.org/10.2106/00004623-200103000-00013

27. Panni AS, Vasso M, Cerciello S. Modular augmentation in revision total knee arthroplasty. Knee Surg Sports Traumatol Arthrosc 2013;21(12):2837-43. https://doi.org/10.1007/s00167-012-2258-1 